\title{
Urinary Concentrations of Metals and Metalloids in Malaysian Adults
}

\author{
Zurahanim Fasha Anual ${ }^{1}$ [ - Noraishah Mohammad Sham ${ }^{1} \cdot$ Rashidah Ambak $^{2} \cdot$ Fatimah Othman $^{3}$. \\ Rafiza Shaharudin ${ }^{1}$
}

Received: 6 July 2020 / Revised: 3 February 2021 / Accepted: 10 March 2021 / Published online: 1 July 2021

(c) The Author(s) 2021

\begin{abstract}
Exposure to environmental pollutants in humans can be conducted through direct measurement of biological media such as blood, urine or hair. Assessment studies of metals and metalloids in Malaysia is very scarce although cross-sectional nationwide human biomonitoring surveys have been established by the USA, Canada, Germany, Spain, France, and Korea. This study aims to assess urinary metal levels namely cadmium (Cd), nickel (Ni), lead ( $\mathrm{Pb}$ ) and arsenic (As) among Malaysian adults. This was a cross-sectional study involving 1440 adults between the age of 18 and 88 years old. After excluding those with $24 \mathrm{~h}$ urine samples of less than $500 \mathrm{ml}$, urine creatinine levels $<0.3 \mathrm{or}>3.0 \mathrm{~g} / \mathrm{L}$ and those who refuse to participate in the study, a total of 817 respondents were included for analysis. A questionnaire with socio-demographic information such as age, gender, occupation, ethnic, academic qualification and medical history was administered to the respondents. Twenty-four-hour urine samples were collected in a container before being transported at $4{ }^{\circ} \mathrm{C}$ to the laboratory. Samples were then aliquoted into $15 \mathrm{ml}$ tubes and kept at $-80^{\circ} \mathrm{C}$ until further analysis. Urine was diluted ten-fold with ultrapure water, filtered and analysed for metals and metalloids using Inductively Coupled Plasma-Mass Spectrometry (ICP-MS). The geometric mean of urinary $\mathrm{As}, \mathrm{Ni}, \mathrm{Cd}$ and $\mathrm{Pb}$ concentrations among adults in Malaysia was 48.21, 4.37, 0.32, and 0.80 $\mu \mathrm{g} / \mathrm{L}$, respectively. Males showed significantly higher urinary metal concentrations compared to females for $\mathrm{As}, \mathrm{Cd}$ and $\mathrm{Pb}$ except for $\mathrm{Ni}$. Those who resided in rural areas exhibited significantly higher $\mathrm{As}, \mathrm{Cd}$ and $\mathrm{Pb}$ urinary concentrations than those who resided in urban areas. As there are no nationwide data on urinary metals, findings from this study could be used to identify high exposure groups, thus enabling policy makers to improve public health strategically.
\end{abstract}

Keywords Biological monitoring $\cdot$ Heavy metals $\cdot$ Arsenic $\cdot$ Cadmium $\cdot$ Lead $\cdot$ General population

\section{Introduction}

Toxic metals and metalloids pose health risk to humans due to their abundance in nature as they are widely deposited in air, soil and water. They have no role in human physiology and may cause intoxication. The most common source of exposure in the general population is through oral intake

Zurahanim Fasha Anual zurahanim@moh.gov.my

1 Environmental Health Research Centre, Institute for Medical Research, National Institutes of Health, Ministry of Health, Shah Alam 40170, Malaysia

2 Centre for Nutrition Epidemiology Research, Institute for Public Health, National Institutes of Health, Ministry of Health, Shah Alam 40170, Malaysia

3 Dietetic and Food Service Department, Hospital Sultanah Aminah, Ministry of Health, Johor Bahru 80000, Malaysia especially from water and food although others may be exposed through occupation or contact with other matrices in the environment (ATSDR 2012). Chronic exposure to metals and metalloids may potentially affect human health in long term and is a cause for concern for health agencies.

Humans may be exposed to multiple forms of arsenic (As), namely, organic and inorganic As. The more toxic form, the inorganic arsenic, is an established human carcinogen and it might also cause non-carcinogenic effects such as developmental retardation, cardiovascular and metabolic disease (IARC 2002; Nava-Acien et al. 2009). The general population are usually exposed to inorganic As from drinking water and eating contaminated food (rice and grains) (EFSA 2009), while exposure to organic As is mainly from consumption of fish and seafood (Fransesconi and Kuehnelt 2004). Cadmium (Cd) is a component of earth's crust, naturally occurring in the environment, as a result of erosion and classified as group 1A carcinogen (ATSDR 2012). Production of nickel-cadmium 
batteries is one of the major source of $\mathrm{Cd}$ in the environment (ATSDR 2012). Other human exposures are from cigarette smoking and contaminated food (soybean, rice, leafy green, nuts and pulses) (EFSA 2009).

Human biomonitoring (HBM) can be defined as "the method for assessing human exposure to chemicals or their effects by measuring these chemicals, their metabolites or reaction products in human specimens" (CDC 2005). HBM is broadly described as measurement or quantification of biomarkers in human biological media such as blood or urine. Regarded as a widely recognized tool which is used to assess and evaluate exposure of general population, population groups, as well as individuals to environmental pollutants, several countries worldwide have successfully conducted large-scale studies and established reference values for metals and metalloids in diverse population. Perhaps, the largest, oldest and long-standing surveillance programme is the National Health and Nutrition Examination Survey (NHANES) in the USA initiated way back in 1971, followed by German Environmental Survey (GerES) in 1985, BIOAMBIENT. ES in Spain (2007) and France (2008) as well as Korea National Survey for Environmental Pollutants in the Human Body (KorSEP) in 2005 (Choi et al. 2017).

While countries from many parts of the globe have initiated human biomonitoring assessment, Malaysia falls short on this as we are yet to establish reference levels of populations exposed to diverse types of chemicals in the environment. To the best of our knowledge, no studies have been conducted thus far assessing pollutants in biological media on a nationwide scale among general population. However, studies pertaining to occupational exposure have been conducted in Malaysia including assessment of heavy metals in toenails of welders (Hariri et al. 2018; Zainal Bakri et al. 2019), cadmium exposure among waste collectors (Ismail et al. 2018), as well as heavy metals levels in nails of farmers (Ahmad Rohi et al. 2012).

Other related HBM studies from other parts of the world such as the European countries managed to identify subjects with an increased level of exposure (as opposed to background exposure) to a given environmental toxin. Using the baseline value of the general population may help toxicologists and physicians identify groups of individuals with higher body burden of the chemicals, thus enabling investigation and control measures to be carried out. In Malaysia, the scarcity of exposure assessment information prompted us to develop this study with the intention to obtain preliminary data on urinary levels of selected metals and metalloids among Malaysian adult population.

\section{Materials and Methods}

\section{Study Design}

This study is a collaboration between Environmental Health Research Centre (EHRC), Institute for Medical Research and Institute for Public Health. Urine samples were obtained from approved project entitled "Populationbased Salt Intake Survey to Support the National Salt Reduction Programme for Malaysia (Malaysian Community Salt Study-MyCoSS)". Since this study is an extension of the MyCoSS study, study design, sample size, sample collection, study instrument and other related parameters adhered to the MyCoSS study (Ambak et al. 2021).

MyCoSS was a cross-sectional population-based household survey. The inclusion criteria for the study were Malaysians aged more than 18 years old, residing in noninstitutional living residences and agreed to participate in the study. Exclusion criteria include pregnant mothers, patients recently began diuretic therapy ( $<4$ weeks), having menses during urine collection, those diagnosed to have chronic diseases (such as kidney disease, heart failure or liver disease) and those having difficulty in collecting urine. To represent the Malaysian population, this survey conducted a stratified cluster sampling method. Sampling design covered both urban and rural areas for every state. Living quarters were randomly selected by the Department of Statistics Malaysia (DOSM). Only one participant was selected from a living quarter. If there were more than one eligible participant, selection was made using a modified Kish Table (Ambak et al. 2021).

This study was conducted between October 2017 and March 2018. Informed consent was obtained from all study participants prior to study commencement. For each subject, the information on age, gender, occupation, education background, health status, income, smoking status, marital status and other relevant information were obtained through a questionnaire which has been pre-tested and validated beforehand. Ethical approval of this study was received from the Medical Research and Ethics Committee [KKM.NIHSEC.P18-795(6)].

\section{Urine Collection and Analysis}

Twenty-four-hour urine samples were collected from participants in clean, metal-free polyethylene containers (without preservatives or stabilizers). The final volume of urine collected for each respondent was recorded at the sampling site by the research team members. The decision to exclude urine samples less than $500 \mathrm{ml}$ for laboratory analysis which were discarded at initial stage was decided 
by the parent study which this study diligently adheres to. Collected samples were transported to a private laboratory at $4{ }^{\circ} \mathrm{C}$. Urine samples were homogenized and then aliquoted for heavy metal analysis and measurement of urine creatinine levels. Aliquoted urine in $15 \mathrm{ml}$ polyethylene containers were sent to our laboratory and kept at $-80{ }^{\circ} \mathrm{C}$ until analysis for metals and metalloids.

Prior to analysis, urine samples were brought to room temperature, thawed, homogenized and diluted 1:10 in the same diluent as the corresponding calibration standards with $2 \%$ nitric acid (suprapur 65\%). Lypochek Urine Metal Control for trace elements Level I and II (Bio-rad) was used for internal quality control. Recoveries for $\mathrm{Ni}, \mathrm{As}, \mathrm{Cd}$, and $\mathrm{Pb}$ were between $85-115 \%$. Observed values fell within the range of certified values. Metals and metalloid determinations were performed using ELAN 9000 ICP-MS (Perkin Elmer) with germanium $(\mathrm{Ge})$, yitrium $(\mathrm{Y})$ and terbium $(\mathrm{Tb})$ used as internal standards. The calibration curves (5 points) were prepared within the concentration ranges normally observed in the general population from overseas studies (Pino et al. 2012; Aprea et al. 2018). The blanks for the analysis were prepared with $2 \%$ nitric acid. Limits of quantification (LOQ) of the blank measures were $0.27 \mu \mathrm{g} / \mathrm{L}$ for As, $0.05 \mu \mathrm{g} / \mathrm{L}$ for $\mathrm{Cd}, 1.40 \mu \mathrm{g} / \mathrm{L}$ for $\mathrm{Ni}$, and $0.60 \mu \mathrm{g} / \mathrm{L}$ for $\mathrm{Pb}$.

\section{Statistical Analysis}

All analyses were performed using IBM SPSS 25. Urinary metal levels were expressed with respect to $\mu \mathrm{g} / \mathrm{g}$ creatinine and log-transformed before analysis. Results with creatinine values below 0.3 and above 3.0 (too low or too high) (WHO 1996) were discarded from analysis. Data were tested for normality prior to analysis using Kolmogorov-Smirnov and Shapiro-Wilk tests. As the data were not normally distributed $(p<0.05)$, results were presented as geometric mean (GM) with 95\% confidence interval. Statistical significance was established at $p<0.05$. Determination of difference between two or more groups was analysed using Kruskal-Wallis test. Limit of detection (LOD) and limit of quantification (LOQ) were calculated as being, respectively, three and ten times the standard deviations of the concentrations of the blank samples. Data below LOD were replaced by value equal to half of the LOQ.

\section{Results}

\section{Characteristics of Study Population}

A total of 1440 adults participated in the study. Of the original sample, 429 individuals were excluded due to refusal of responding to questionnaire $(n=138)$, not a Malaysian resident $(n=35)$, sick $(n=16)$ and residence were locked during survey $(n=240)$. Another 194 individuals were excluded for reasons such as urine samples of less than $500 \mathrm{ml}(n=137)$ (Land et al. 2014), urine creatinine levels of $<0.3 \mathrm{~g} / \mathrm{L}$ or $>3.0 \mathrm{~g} / \mathrm{L}$ (WHO 1996) $(n=37)$, and cracked sample tubes $(n=20)$, leaving 817 individuals for present analysis. Although a total of 623 individuals were excluded from analysis, the findings did not differ from those included in the study.

The socio-demographics of respondents in this study was described and categorized by gender, age group, ethnic, education level, occupation, residence, smoking habit and Body Mass Index (BMI) as shown in Table 1. The number of male respondents was $346(42.4 \%)$, while the female respondents were 471 (57.6\%). Majority of respondents were above 45 years of age for both males: $64(7.8 \%)$ and females 110 (13.5\%). Most of the study population was Malay (64\%), followed by Chinese (10.5\%) and Indian (5.9\%) which is coherent to ethnic distribution in Malaysia (DOSM 2020), predominantly being housewives (28.3\%), possessed secondary education $(61.8 \%)$, chiefly resided in rural (58\%) as opposed to urban (42\%), non-smokers $(67.6 \%)$ and had an average BMI of $26.7 \mathrm{~kg} / \mathrm{m}^{2}( \pm 5.3)$.

\section{Distribution of Metals and Metalloid in Urine}

Table 2 and 3 present the urinary metals results in $\mu \mathrm{g} / \mathrm{L}$ and $\mu \mathrm{g} / \mathrm{g}$ creatinine. The geometric mean of urinary metals concentrations among adults in Malaysia was As $(48.21 \mu \mathrm{g} / \mathrm{L}$; 95\% CI: 44.82-51.83), Ni (4.37 $\mu \mathrm{g} / \mathrm{L}$; 95\% CI: 4.15-4.61), $\mathrm{Cd}(0.32 \mu \mathrm{g} / \mathrm{L} ; 95 \% \mathrm{CI}: 0.30-0.34)$ and $\mathrm{Pb}(0.80 \mu \mathrm{g} / \mathrm{L} ; 95 \%$ CI: 0.74-0.86). Urinary geometric mean was significantly higher in males as opposed to females for As $(54.11 \mu \mathrm{g} / \mathrm{L}$; 95\% CI: 48.28-60.65, $p<0.003), \mathrm{Cd}(0.37 \mu \mathrm{g} / \mathrm{L} ; 95 \% \mathrm{CI}$ : $0.34-0.41, p<0.000)$ and $\mathrm{Pb}(0.97 \mu \mathrm{g} / \mathrm{L}$; 95\% CI: 0.86-1.09, $p<0.000)$. Smoking respondents showed significantly elevated urinary levels for $\mathrm{Ni}(4.73 \mu \mathrm{g} / \mathrm{L}$; 95\% CI: 4.29-5.21, $p<0.025), \mathrm{Cd}(0.36 \mu \mathrm{g} / \mathrm{L} ; 95 \%$ CI: $0.32-0.41, p<0.007)$ and $\mathrm{Pb}(1.02 \mu \mathrm{g} / \mathrm{L} ; 95 \% \mathrm{CI}: 0.90-1.17 p<0.000)$, but not for As. Those who reside in rural areas exhibited significantly higher As $(51.60 \mu \mathrm{g} / \mathrm{L} ; 95 \% \mathrm{CI}: 46.93-56.75, p<0.015)$, $\mathrm{Cd}(0.34 \mu \mathrm{g} / \mathrm{L} ; 95 \% \mathrm{CI}: 0.27-0.33, p<0.044)$, and $\mathrm{Pb}$ $(0.34 \mu \mathrm{g} / \mathrm{L} ; 95 \% \mathrm{CI}: 0.31-0.37, p<0.036)$ urinary concentrations than those who reside in urban areas. The urinary concentrations were significantly different among respondents from various age groups only for $\mathrm{Cd}(p<0.002)$, with respondents above 65 years of age showing the highest levels in females compared to males for As and Cd (Figs. 1, 2, 3, 4).

\section{Comparison of Urinary Metals Concentrations Between Countries}

The 95th percentile values for metals from this study were compared with values observed from international studies, 
Table 1 Demographic characteristics of Malaysian by total population and gender

\begin{tabular}{|c|c|c|c|}
\hline & Total N (\%) & Men N (\%) & Women N (\%) \\
\hline All & $817(100)$ & $346(42.4)$ & $471(57.6)$ \\
\hline \multicolumn{4}{|l|}{ Age group (years) } \\
\hline Mean \pm S.D & $48.9 \pm 15.2$ & $49.6(15.7)$ & $48.4(14.8)$ \\
\hline $18-24$ & $55(6.7)$ & $24(2.9)$ & $31(3.8)$ \\
\hline $25-34$ & $123(15.1)$ & $53(6.5)$ & $70(8.6)$ \\
\hline $35-44$ & $134(16.4)$ & $49(6.0)$ & $85(10.4)$ \\
\hline $45-54$ & $174(21.3)$ & $64(7.8)$ & $110(13.5)$ \\
\hline $55-64$ & $193(23.6)$ & $91(11.1)$ & $102(12.5)$ \\
\hline$>65$ & $138(16.9)$ & $65(8.0)$ & $73(8.9)$ \\
\hline \multicolumn{4}{|l|}{ Ethnic } \\
\hline Malay & $522(64.0)$ & $224(27.4)$ & $298(36.5)$ \\
\hline Chinese & $86(10.5)$ & $33(4.0)$ & $53(6.5)$ \\
\hline Indian & $48(5.9)$ & $13(1.6)$ & $35(4.3)$ \\
\hline Bumiputera Sabah/Sarawak & $150(18.4)$ & $69(8.4)$ & $81(9.9)$ \\
\hline Others & $10(1.2)$ & $6(0.7)$ & $4(0.5)$ \\
\hline \multicolumn{4}{|l|}{ Education level } \\
\hline Primary & $173(21.2)$ & $76(9.3)$ & 97 (11.9) \\
\hline Secondary & $505(61.8)$ & $211(25.8)$ & $294(36.0)$ \\
\hline Tertiary & $79(9.7)$ & $42(5.1)$ & $37(4.5)$ \\
\hline None & $60(7.3)$ & $17(2.1)$ & $43(5.3)$ \\
\hline \multicolumn{4}{|l|}{ Occupation } \\
\hline Public sector & $120(14.7)$ & $65(8.0)$ & $55(6.7)$ \\
\hline Private sector & $126(15.4)$ & $71(8.7)_{-}$ & $55(6.7)$ \\
\hline Housewives & $231(28.3)$ & $0(0)$ & $231(28.3)$ \\
\hline Self-employed & $180(22.0)$ & $111(13.6)$ & $69(8.4)$ \\
\hline Student & $14(1.7)$ & $8(1.0)$ & $6(0.7)$ \\
\hline Unemployed & $111(13.6)$ & $64(7.8)$ & $47(5.8)$ \\
\hline Others & $35(4.3)$ & $27(3.3)$ & $8(1)$ \\
\hline Residence & $343(42.0)$ & $146(17.9)$ & $197(24.1)$ \\
\hline Urban & $474(58.0)$ & $200(24.5)$ & $274(33.5)$ \\
\hline \multicolumn{4}{|l|}{ Rural } \\
\hline Smoking habit & $552(67.6)$ & $108(13.2)$ & $444(54.3)$ \\
\hline Non-smoker & $265(32.4)$ & $238(29.1)$ & $27(3.3)$ \\
\hline Smoker & $160(19.6)$ & $151(18.5)$ & $9(1.1)$ \\
\hline \multicolumn{4}{|l|}{ Former smoker } \\
\hline \multicolumn{4}{|l|}{ Body Mass Index (BMI-kg/m²) } \\
\hline Mean \pm S.D & $26.7 \pm 5.3$ & $25.9 \pm 4.8$ & $27.3 \pm 5.7$ \\
\hline$<18.5$ (Underweight) & $35(4.3)$ & $17(4.9)$ & $18(3.9)$ \\
\hline 18.5-24.9 (Normal weight) & $288(35.3)$ & $132(38.1)$ & $156(33.1)$ \\
\hline 24.9-29.9 (Overweight) & $298(36.5)$ & $135(39.0)$ & $163(34.6)$ \\
\hline$>30($ Obese $)$ & $196(23.9)$ & $62(18)$ & $134(28.4)$ \\
\hline
\end{tabular}

namely, Germany, France, Flanders, USA, Canada, and South Korea as shown in Table 4. Results from this study showed that the 95th percentile values for urinary arsenic in Malaysian adult populations was found to be the highest between 2.2 times higher than adults in South Korea (Lee et al. 2012) to 17 times higher than adults in Germany (Wilhelm et al. 2004). Urinary Ni 95th percentile values in
Malaysian adults were about 3 times higher than those in Belgium and Canada. On the other hand, urinary $\mathrm{Cd}$ 95th percentile value in Malaysia were comparable with Canadian adult population and seem to be in the intermediate range between the lowest urinary $\mathrm{Cd}$ concentrations in Flanders and the highest urinary $\mathrm{Cd}$ concentrations in South Korea. 
Table 2 Urinary metal concentrations levels $(\mu \mathrm{g} / \mathrm{L})$ in Malaysia by total population, gender, smoking status and strata

\begin{tabular}{|c|c|c|c|c|c|c|c|c|c|c|}
\hline & \multirow[t]{2}{*}{$\mathrm{N}$} & \multirow[t]{2}{*}{$\mathrm{AM}$} & \multirow[t]{2}{*}{$\mathrm{Sd}$} & \multirow[t]{2}{*}{ GM } & \multirow{2}{*}{$\begin{array}{l}\text { 95\% CI } \\
\text { (lower limits, } \\
\text { upper limits) }\end{array}$} & \multicolumn{5}{|c|}{ Percentile } \\
\hline & & & & & & 25 th & 50th & 75th & 90th & 95th \\
\hline \multicolumn{11}{|l|}{ Arsenic (As) } \\
\hline Total & 817 & 82.33 & 105.03 & 48.21 & $44.82,51.83$ & 24.14 & 47.76 & 103.06 & 194.81 & 259.48 \\
\hline Male & 346 & 90.42 & 98.06 & 54.11 & $48.28,60.65$ & 27.62 & 55.06 & 124.05 & 221.26 & 283.20 \\
\hline Female & 471 & 76.38 & 109.58 & 44.28 & $40.32,48.63$ & 22.69 & 43.54 & 92.30 & 162.37 & 238.23 \\
\hline Non-smoker & 552 & 79.18 & 108.78 & 45.90 & $42.03,50.14$ & 23.80 & 45.58 & 94.58 & 175.21 & 252.92 \\
\hline Smoker & 265 & 88.87 & 96.60 & 53.37 & $46.95,60.65$ & 26.70 & 54.29 & 123.50 & 221.56 & 271.09 \\
\hline Urban & 343 & 77.51 & 115.00 & 43.86 & $39.19,49.09$ & 23.78 & 39.36 & 98.97 & 182.68 & 244.23 \\
\hline Rural & 474 & 85.81 & 97.14 & 51.60 & $46.93,56.75$ & 25.81 & 54.11 & 104.62 & 212.13 & 280.03 \\
\hline \multicolumn{11}{|l|}{ Nickel (Ni) } \\
\hline Total & 817 & 5.73 & 4.34 & 4.37 & $4.15,4.61$ & 2.77 & 4.49 & 7.51 & 11.51 & 13.93 \\
\hline Male & 346 & 6.17 & 4.86 & 4.57 & $4.19,4.99$ & 2.76 & 4.78 & 8.17 & 12.25 & 14.94 \\
\hline Female & 471 & 5.41 & 3.89 & 4.24 & $3.96,4.53$ & 2.78 & 4.16 & 7.01 & 10.97 & 13.40 \\
\hline Non-smoker & 552 & 5.46 & 4.03 & 4.21 & $3.96,4.49$ & 2.71 & 4.26 & 7.07 & 11.15 & 13.31 \\
\hline Smoker & 265 & 6.29 & 4.89 & 4.73 & $4.29,5.21$ & 2.87 & 4.92 & 8.04 & 12.26 & 15.13 \\
\hline Urban & 343 & 5.56 & 4.38 & 4.11 & $3.78,4.49$ & 2.58 & 4.30 & 7.35 & 11.53 & 14.13 \\
\hline Rural & 474 & 5.85 & 4.31 & 4.57 & $4.28,4.88$ & 2.92 & 4.61 & 7.71 & 11.52 & 13.89 \\
\hline \multicolumn{11}{|l|}{ Cadmium (Cd) } \\
\hline Total & 817 & 0.47 & 0.43 & 0.32 & $0.30,0.34$ & 0.19 & 0.35 & 0.62 & 1.04 & 1.34 \\
\hline Male & 346 & 0.54 & 0.49 & 0.37 & $0.34,0.41$ & 0.22 & 0.42 & 0.67 & 1.14 & 1.55 \\
\hline Female & 471 & 0.43 & 0.37 & 0.29 & $0.26,0.31$ & 0.17 & 0.31 & 0.59 & 0.96 & 1.21 \\
\hline Non-smoker & 552 & 0.44 & 0.38 & 0.30 & $0.28,0.33$ & 0.17 & 0.32 & 0.59 & 0.96 & 1.22 \\
\hline Smoker & 265 & 0.54 & 0.51 & 0.36 & $0.32,0.41$ & 0.21 & 0.38 & 0.70 & 1.15 & 1.58 \\
\hline Urban & 343 & 0.43 & 0.36 & 0.30 & $0.27,0.33$ & 0.18 & 0.32 & 0.59 & 0.90 & 1.16 \\
\hline Rural & 474 & 0.51 & 0.47 & 0.34 & $0.31,0.37$ & 0.19 & 0.37 & 0.64 & 1.16 & 1.43 \\
\hline \multicolumn{11}{|l|}{ Lead $(\mathrm{Pb})$} \\
\hline Total & 817 & 1.53 & 2.21 & 0.80 & $0.74,0.86$ & 0.28 & 0.70 & 1.89 & 3.93 & 5.75 \\
\hline Male & 346 & 1.75 & 2.13 & 0.97 & $0.86,1.09$ & 0.28 & 0.96 & 2.30 & 4.17 & 6.06 \\
\hline Female & 471 & 1.38 & 2.26 & 0.69 & $0.63,0.76$ & 0.28 & 0.28 & 1.43 & 3.51 & 5.45 \\
\hline Non-smoker & 552 & 1.38 & 2.16 & 0.71 & $0.65,0.77$ & 0.28 & 0.58 & 1.55 & 3.50 & 5.39 \\
\hline Smoker & 265 & 1.86 & 2.29 & 1.02 & $0.90,1.17$ & 0.28 & 1.11 & 2.45 & 4.17 & 6.33 \\
\hline Urban & 343 & 1.71 & 2.39 & 0.30 & $0.27,0.33$ & 0.28 & 0.80 & 2.31 & 4.32 & 6.20 \\
\hline Rural & 474 & 1.45 & 2.07 & 0.34 & $0.31,0.37$ & 0.28 & 0.66 & 1.64 & 3.37 & 5.35 \\
\hline
\end{tabular}

AM arithmetic mean, Sd standard deviation, GM geometric mean, GSD geometric standard deviation, 95\% CI95\% confidence interval

\section{Discussion}

This study aimed to determine the urinary $\mathrm{As}, \mathrm{Ni}, \mathrm{Cd}$, and $\mathrm{Pb}$ levels among adults in Malaysia. To the best of our knowledge, this is the first study which successfully quantified metal levels in urine of Malaysian respondents on a national scale. This baseline information will contribute to identify Malaysian adults with high exposure group between 18 and 88 years of age. The discrepancies in values reported in other parts of the world might be explained by diverse environmental exposure profiles as well as unique dietary patterns of a particular nation. This is apparent in several studies conducted worldwide, as the values in this study are similar to those reported in certain areas, but could also be lower or higher depending on the vicinity of the study location.

It was reported that per capita fish consumptions by Malaysian adults was $54.77 \mathrm{~kg} /$ person/year in the year 2016, making it the second highest among Asian nations, after Japan (Nurul et al. 2016). Discrepancies between average per capita fish consumption exist and are considerably different across and within countries and regions due to the influence of culture, economy and geographical factors (FAO 2018). The fact that Malaysians are high fish consumers' with the Malays significantly consuming fish higher than Chinese and Indians (Nurul et al. 2016) may well respond to the relatively high urinary total arsenic concentrations at 95 th percentile 
Table 3 Creatinine-corrected metal concentrations $(\mu \mathrm{g} / \mathrm{g})$ in Malaysian population

\begin{tabular}{|c|c|c|c|c|c|c|c|c|c|c|}
\hline & \multirow[t]{2}{*}{$\mathrm{N}$} & \multirow[t]{2}{*}{$\mathrm{AM}$} & \multirow[t]{2}{*}{ S.D } & \multirow[t]{2}{*}{ GM } & \multirow{2}{*}{$\begin{array}{l}\text { 95\% CI } \\
\text { (lower limits, } \\
\text { upper limits) }\end{array}$} & \multicolumn{5}{|c|}{ Percentile } \\
\hline & & & & & & 25 th & 50 th & 75 th & 90th & 95 th \\
\hline \multicolumn{11}{|l|}{ Arsenic (As) } \\
\hline Total & 817 & 93.63 & 145.29 & 52.67 & $48.94,56.69$ & 25.94 & 51.00 & 105.85 & 207.78 & 313.91 \\
\hline Male & 346 & 78.33 & 92.76 & 45.62 & $40.67,51.18$ & 22.92 & 46.02 & 95.87 & 178.44 & 271.27 \\
\hline Female & 471 & 104.87 & 173.30 & 58.55 & $53.27,64.36$ & 28.66 & 57.16 & 109.19 & 235.99 & 325.69 \\
\hline Non-smoker & 552 & 100.05 & 163.47 & 55.20 & $50.41,60.44$ & 27.03 & 54.94 & 107.52 & 234.64 & 316.45 \\
\hline Smoker & 265 & 80.25 & 95.93 & 47.80 & $42.19,54.14$ & 24.34 & 44.57 & 100.53 & 185.82 & 277.65 \\
\hline Urban & 343 & 85.32 & 144.31 & 46.91 & $41.95,52.47$ & 22.85 & 44.57 & 91.14 & 176.20 & 297.65 \\
\hline Rural & 474 & 99.64 & 145.84 & 57.29 & $52.00,63.12$ & 27.90 & 60.93 & 115.98 & 225.22 & 317.10 \\
\hline \multicolumn{11}{|l|}{ Nickel (Ni) } \\
\hline Total & 817 & 6.41 & 5.04 & 4.78 & $4.52,5.05$ & 3.05 & 5.15 & 8.22 & 13.24 & 17.16 \\
\hline Male & 346 & 5.26 & 4.31 & 3.85 & $3.53,4.21$ & 2.29 & 4.26 & 6.63 & 10.33 & 13.42 \\
\hline Female & 471 & 7.26 & 5.36 & 5.60 & $5.22,6.00$ & 3.61 & 5.72 & 9.57 & 14.46 & 18.26 \\
\hline Non-smoker & 552 & 6.76 & 5.06 & 5.06 & $4.73,5.42$ & 3.21 & 5.40 & 8.80 & 14.14 & 17.76 \\
\hline Smoker & 265 & 5.68 & 4.91 & 4.23 & $3.84,4.66$ & 2.88 & 4.64 & 7.00 & 10.81 & 14.41 \\
\hline Urban & 343 & 6.12 & 4.89 & 4.40 & $4.00,4.83$ & 2.85 & 5.02 & 8.03 & 13.12 & 17.48 \\
\hline Rural & 474 & 6.62 & 5.13 & 5.07 & $4.73,5.43$ & 3.20 & 5.30 & 8.25 & 13.56 & 16.68 \\
\hline \multicolumn{11}{|l|}{ Cadmium (Cd) } \\
\hline Total & 817 & 0.53 & 0.50 & 0.35 & $0.33,0.37$ & 0.20 & 0.38 & 0.68 & 1.20 & 1.60 \\
\hline Male & 346 & 0.47 & 0.48 & 0.31 & $0.28,0.35$ & 0.18 & 0.34 & 0.58 & 0.98 & 1.48 \\
\hline Female & 471 & 0.57 & 0.52 & 0.38 & $0.35,0.42$ & 0.22 & 0.40 & 0.79 & 1.29 & 1.65 \\
\hline Non-smoker & 552 & 0.55 & 0.49 & 0.36 & $0.33,0.40$ & 0.20 & 0.39 & 0.77 & 1.26 & 1.64 \\
\hline Smoker & 265 & 0.49 & 0.52 & 0.32 & $0.29,0.36$ & 0.18 & 0.36 & 0.59 & 0.99 & 1.52 \\
\hline Urban & 343 & 0.47 & 0.44 & 0.32 & $0.29,0.35$ & 0.19 & 0.34 & 0.59 & 1.07 & 1.55 \\
\hline Rural & 474 & 0.57 & 0.54 & 0.38 & $0.35,0.41$ & 0.20 & 0.41 & 0.79 & 1.25 & 1.65 \\
\hline \multicolumn{11}{|l|}{ Lead $(\mathrm{Pb})$} \\
\hline Total & 817 & 1.70 & 2.79 & 0.87 & $0.81,0.94$ & 0.35 & 0.76 & 1.88 & 4.29 & 6.28 \\
\hline Male & 346 & 1.53 & 2.01 & 0.82 & $0.72,0.92$ & 0.26 & 0.85 & 1.88 & 3.63 & 5.36 \\
\hline Female & 471 & 1.83 & 3.25 & 0.92 & $0.83,1.01$ & 0.37 & 0.71 & 1.87 & 4.74 & 6.54 \\
\hline Non-smoker & 552 & 1.70 & 2.99 & 0.85 & $0.78,0.93$ & 0.35 & 0.67 & 1.82 & 4.42 & 6.31 \\
\hline Smoker & 265 & 1.71 & 2.33 & 0.92 & $0.80,1.05$ & 0.31 & 0.98 & 2.08 & 3.86 & 6.19 \\
\hline Urban & 343 & 1.83 & 3.19 & 0.94 & $0.84,1.10$ & 0.39 & 0.85 & 2.07 & 4.82 & 6.09 \\
\hline Rural & 474 & 1.61 & 2.46 & 0.83 & $0.75,0.91$ & 0.33 & 0.71 & 1.85 & 3.91 & 6.35 \\
\hline
\end{tabular}

AM arithmetic mean, Sd standard deviation, GM geometric mean, GSD geometric standard deviation, 95\% CI95\% confidence interval

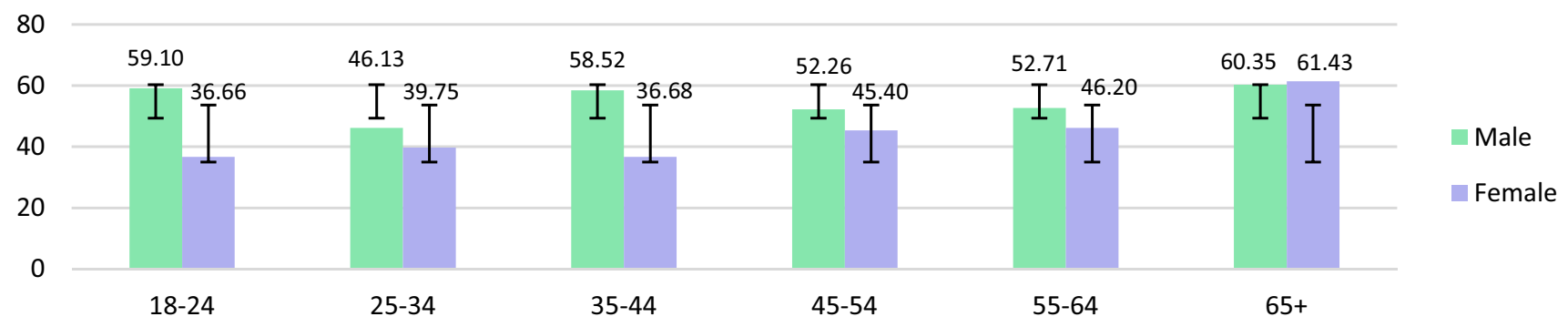

Fig. 1 Urinary As concentrations ( $\mu \mathrm{g} / \mathrm{L})$ between males and females of different age categories (Note: error bars represent standard deviation)

$(259.48 \mu \mathrm{g} / \mathrm{L})$. Seafood intake markedly increase urine concentrations of total As as well as other As species (Choi et al.
2010; Navas-Acien et al. 2011) and respondents eating fish within 3 days showed significantly higher urinary As levels 


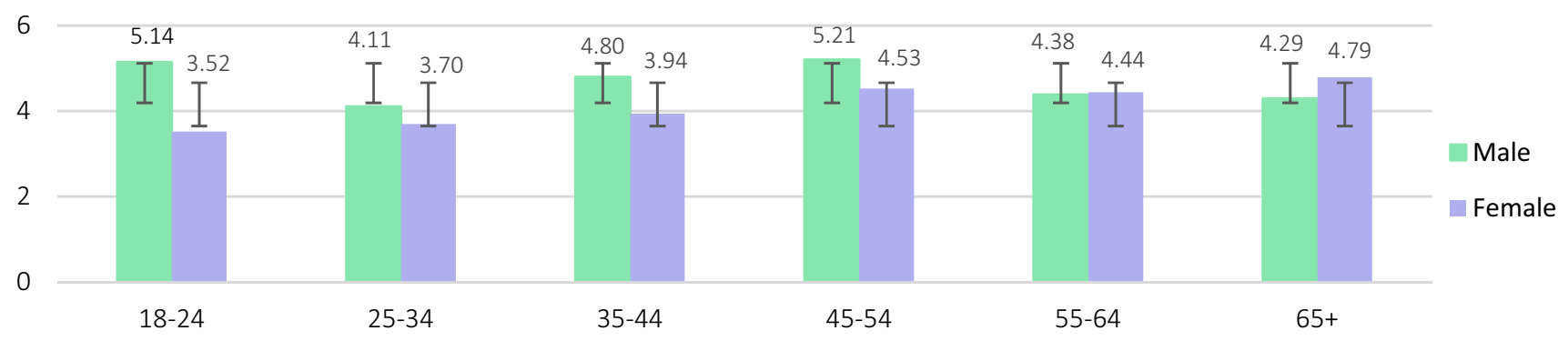

Fig. 2 Urinary Ni concentrations ( $\mu \mathrm{g} / \mathrm{L}$ ) between males and females of different age categories

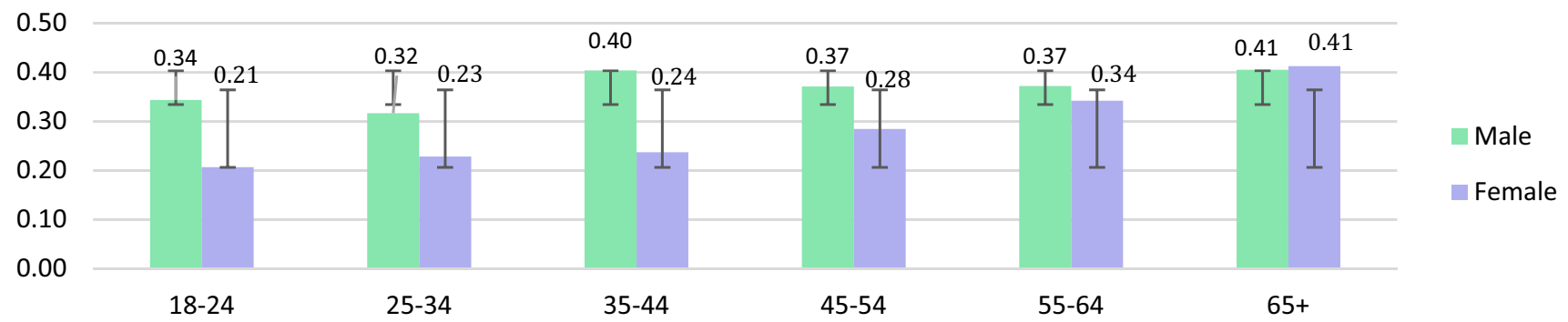

Fig. 3 Urinary Cd concentrations $(\mu \mathrm{g} / \mathrm{L})$ between males and females of different age categories

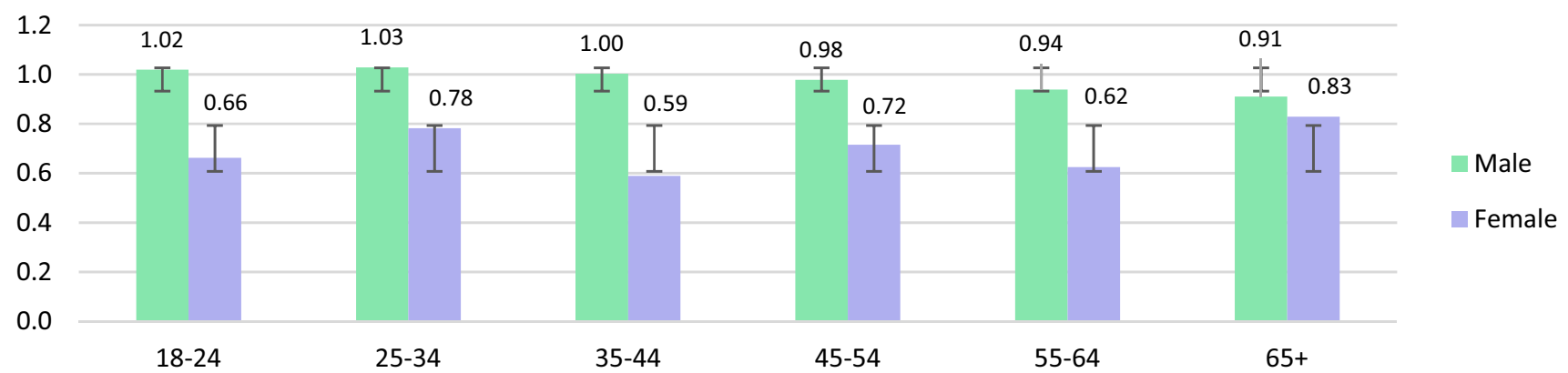

Fig. 4 Urinary $\mathrm{Pb}$ concentrations ( $\mu \mathrm{g} / \mathrm{L})$ between males and females of different age categories

than those who did not (Lee et al. 2012). Other researchers also have established associations between urinary As excretion and consumption of seafood (Becker et al. 2003; Aguilera et al. 2008).

It is worth to note that toxicity of As largely depends on its oxidation states thus arsenic toxicity may be inaccurately assessed when analyses are restricted to total As alone. Speciation analysis is essential to distinguish the toxic and non-toxic fractions of the arsenic in specific media. Studies have shown that the proportion of arsenobetaine (organic As) in fish may be between 50\% (Cubadda et al. 2017) and $87 \%$ (Lin et al. 2008) hence toxicity of dietary As exposure may have been overestimated if As in fish is presumed to be present exclusively as inorganic As. Inorganic As; the highly toxic form of arsenics are metabolized in the body as either monomethylarsonate (MMA) or dimethylarsonate (DMA)(Aposhian \& Aposhian 2006). The ability of the body to metabolize either to MMA or
DMA is profound to human health as MMA is linked to cause cancers and cardiovascular problems (Steinmaus et al. 2006; Wu et al. 2006).

In this study, the creatinine-adjusted urinary levels of As, $\mathrm{Ni}, \mathrm{Cd}$, and $\mathrm{Pb}$ were significantly higher in females than males. The patterns of occurrence by gender is similar to those reported in Italy for $\mathrm{Ni}$ (Aprea et al. 2018), Spain for Cd (López-Herranz et al. 2015) as well as in Korea for As (Park et al. 2016) by which the results might be influenced by the values of urinary creatinine. Due to higher muscle mass in males (Barr et al. 2005), creatinine concentrations are relatively higher for males than for females (Cocker et al. 2011). Differences in diet, behaviour and metabolism may explain the effects of gender on urinary metals levels (Arbuckle 2006) and the sex-related differences may also be influenced by physiological factors such as menstrual and reproductive factors (Lee and Kim 2014). 
Table 4 Comparison of urinary metals concentrations between countries

\begin{tabular}{|c|c|c|c|c|c|}
\hline Country & Metals & 95th percentile & Study population & Study period & Reference \\
\hline $\begin{array}{l}\text { Germany } \\
\text { Belgium }\end{array}$ & As & $\begin{array}{l}15.0 \mu \mathrm{g} / \mathrm{L} \\
48.8 \mu \mathrm{g} / \mathrm{L}\end{array}$ & $\begin{array}{l}\text { Adults (age 18-69) } \\
\text { Adults (age } \geq 18 \text { ) }\end{array}$ & $\begin{array}{l}1997-1999 \\
2010-2011\end{array}$ & $\begin{array}{l}\text { Wilhelm et al. (2004) } \\
\text { Hoet et al. (2013) }\end{array}$ \\
\hline Flanders & & $90.0 \mu \mathrm{g} / \mathrm{L}$ & Adults (age 20-40) & 2007-2011 & Schoeters et al. (2012) \\
\hline USA & & $52.4 \mu \mathrm{g} / \mathrm{L}$ & Males and females $($ age $\geq 6$ ) & 2011-2012 & CDC (2009) \\
\hline Canada & & $76.0 \mu \mathrm{g} / \mathrm{L}$ & Canadian population (age 3-79) & 2009-2011 & CHMS (2013) \\
\hline South Korea & & $119.7 \mu \mathrm{g} / \mathrm{L}$ & Adults (age $\geq 20$ ) & 2008 & Lee et al. (2012) \\
\hline Malaysia & & $260.0 \mu \mathrm{g} / \mathrm{L}$ & Adults (age 18-88) & $2017-2018$ & This study \\
\hline Belgium & $\mathrm{Ni}$ & $4.73 \mu \mathrm{g} / \mathrm{L}$ & Adults (age $\geq 18$ ) & $2010-2011$ & Hoet et al. (2013) \\
\hline Canada & & $4.80 \mu \mathrm{g} / \mathrm{L}$ & Canadian population (age 3-79) & 2009-2011 & CHMS (2013) \\
\hline Malaysia & & $15.0 \mu \mathrm{g} / \mathrm{L}$ & Adults (age 18-88) & $2017-2018$ & This study \\
\hline Flanders & $\mathrm{Cd}$ & $0.44 \mu \mathrm{g} / \mathrm{L}$ & Non-smoking adults (age 18-69) & 1997-1999 & Schoeters et al. (2012) \\
\hline USA & & $0.87 \mu \mathrm{g} / \mathrm{L}$ & Males and females (age $\geq 6$ ) & 2011-2012 & CDC (2009) \\
\hline Canada & & $1.80 \mu \mathrm{g} / \mathrm{L}$ & Canadian population (age 3-79) & 2009-2011 & CHMS (2013) \\
\hline South Korea & & $3.11 \mu \mathrm{g} / \mathrm{L}$ & Adults (age $\geq 20$ ) & 2008 & Lee et al. (2012) \\
\hline Malaysia & & $1.50 \mu \mathrm{g} / \mathrm{L}$ & Adults (age 18-88) & 2017-2018 & This study \\
\hline Canada & $\mathrm{Pb}$ & $1.90 \mu \mathrm{g} / \mathrm{L}$ & Canadian population (age 3-79) & 2009-2011 & CHMS (2013) \\
\hline Malaysia & & $6.00 \mu \mathrm{g} / \mathrm{L}$ & Adults (age 18-88) & 2017-2018 & This study \\
\hline
\end{tabular}

This study observed urinary $\mathrm{As}, \mathrm{Ni}, \mathrm{Cd}$, and $\mathrm{Pb}$ levels which were higher in smokers rather than non-smokers. Studies by other researchers (Mortada et al. 2002; LópezHerranz et al. 2015) revealed that subjects who were smokers had higher urinary $\mathrm{Cd}$ levels compared to subjects who did not smoke; thereby suggesting tobacco as main source of exposure in the general population (Jarup and Akesson 2009). Former and current smokers usually exhibit higher urinary cadmium levels as opposed to never-smokers (Olsson et al. 2002).

Taking age into account, urinary $\mathrm{As}, \mathrm{Cd}$, and $\mathrm{Pb}$ were significantly higher in males as opposed to females $(p<0.05)$ although no evident trend was observed between the males' age categories. Females however showed upward trend for As, $\mathrm{Ni}$, and $\mathrm{Cd}$ among those from different age categories while female respondents above 65 years old had the highest urinary As and Cd concentrations. Aprea et al. (2018) exhibited similar trends for urinary $\mathrm{Cd}$ levels in subjects above 40 years old as opposed to younger subjects. Some metals such as $\mathrm{Cd}$ and $\mathrm{Pb}$ may accumulate with age which may explain the higher urinary metals levels in the elder participants compared to the younger ones (Yasuda et al. 2012).

As biomonitoring studies in South East Asia is very scarce, established reference ranges from European and Asian population were used for comparison. Although these comparisons are quite biased as differences exist in terms of environmental exposures, geographical locations, eating habits, lifestyles and others, they do provide a benchmark for comparison among the observed elements of interest. Reference values as defined by the Commission on Human Biological Monitoring of the German Federal Environmental Agency is "intended to characterize the upper margin of the current background exposure of the general population to a given environmental toxin at a given time". Reference values are strictly statistically derived values which is used to establish guideline values for population with increased internal exposure to environmental toxin and cannot be used to evaluate health-related criteria (Ewers et al. 1999).

On the other hand, human biomonitoring values (HBM) originated from toxicological and epidemiological studies hence can be used to represent health-related biological exposure limits by which two levels (HBM I \& HBM II) were recommended by the commission. HBM I is the concentration of environmental toxin in biological media which pose no risk for adverse health effects to individuals in the general population (Ewers et al. 1999). HBM II is the concentration of environmental toxin in biological media which pose increased risk for adverse health effects in susceptible individuals of the general population (Ewers et al. 1999).

The health-based value for $\mathrm{Cd}$ in urine established by the German Human Biomonitoring Commission is $1 \mu \mathrm{g} / \mathrm{L}$ by which there is no risk for adverse health effects (HBM I) (Schulz et al. 2011). In this study, $10.5 \%$ of participants had Cd levels exceeding the HBM I levels suggesting that this population might be at risk as $\mathrm{Cd}$ is a recognized human carcinogen (IARC, category 1) and a well-known nephrotoxic agent. Low exposure to urinary $\mathrm{Cd}$ levels in the general population have shown substantial transformation in biomarkers for early kidney damage (Akesson et al. 2005; Noonan et al. 2002). Similar to As and Ni, urinary 95th percentile of $\mathrm{Pb}$ $\mathrm{RV}_{95}$ is about 3 times higher than that of the Canadians.

The 95th percentile for Malaysia was the highest for urinary $\mathrm{As}, \mathrm{Ni}$, and $\mathrm{Pb}$ except for $\mathrm{Cd}$. Since this is the first biomonitoring study which is conducted in Malaysia, it 
is expected that the findings will be on the higher end of the spectrum. HBM data from other countries in Europe and Asia have seen reduction in levels of pollutants since the first HBM was initiated. This is possible as the available biomonitoring data was acted upon and measures are implemented to reduce exposure among the general population after series of HBM surveys. For instance, the US Environmental Protection Agency (EPA) has set the maximum arsenic level in public water systems at $50 \mu \mathrm{g} / \mathrm{L}$ for decades and in January 2006, the level was set to $10 \mu \mathrm{g} / \mathrm{L}$. A study by Nigra et al. (2003) showed that the implementation of the new regulation has seen reductions in drinking water arsenic exposure among public well water users thereby confirming vital role of federal drinking water regulations in reducing toxic exposures and protecting human health. On the other hand, the findings from this study will provide stakeholders and policy makers with urinary metals and metalloids data hence appropriate risk reduction strategies could be executed.

This research has some limitations. First, the ratios of age and gender were greatly skewed in this study. The study population was mostly females and majority of the respondents were more than 45 years old. The data collection more often than not was conducted during working hours therefore mostly housewives were available as well as the elderly. Second, this study measures only total As concentrations in urine instead of As speciation. Thus, interpretation of As in urine may be less accurate and overrated as fraction of organic As (non-toxic) is usually more than inorganic As (toxic). Third, details on fish consumption from the respondents were not available hence only assumptions could be made that the respondents with high urinary As concentrations may have eaten fish or seafood prior to $24 \mathrm{~h}$ urine sampling. Fourth, this study measures urine; which reflects current exposure rather than hair or nails which could reflect long-term exposure in humans. Fifth, collection of $24 \mathrm{~h}$ urine samples was a cumbersome process as it critically affects response rate. Volume of urine sample less than $500 \mathrm{ml}$ was decided to be discarded by the parent study therefore no urine samples were received for that particular individuals. As aforementioned, samples included for analysis continues to be representative of the original study sample. Hence, future study should consider collection of spot urine samples instead of $24 \mathrm{~h}$ urine samples. Lastly, comparison between findings from this study and other studies in Malaysia is very challenging as other studies measured either urine in occupationally exposed population or trace elements concentrations in hair.

\section{Conclusions}

In conclusion, the urinary concentrations of arsenic, nickel, cadmium and lead obtained from this study provided information on urine metals and metalloid levels in Malaysian adults. This study measured population body burden in adults and evaluated effects of gender, age and smoking status influencing urinary exposure. It should be noted, however, that this study did not conduct arsenic speciation; hence future studies should include measurement of inorganic and organic species to differentiate the proportion of toxic and non-toxic fraction which are mainly derived from fish and seafood consumption. In addition, future studies should also include respondents' information on fish and seafood consumption or exclude those who eat fish and seafood entirely from being study subject. Subjects younger than 18 years old should be included as study subjects to enable a more comprehensive biomonitoring data.

Acknowledgements The authors would like to thank the Director General of Health, Malaysia for the permission to publish this article. The study was supported by the Ministry of Health (MOH). The MOH had no role in the design and conduct of the study; in the collection, analyses, or interpretation of data; in the writing of the manuscript or in the decision to publish the results. We would like to thank Mrs Rashidah Ambak and Mrs Fatimah Othman for assistance in urine sample collection. Our deepest gratitude goes to Mrs Cathrinena Anak Robun and $\mathrm{Mr}$ Mohamad Hairulhisam bin Hairi (EHRC, IMR) for assisting in sample analysis throughout the commencement of the project.

\section{Declarations}

Conflict of interest The authors declare no conflict of interest.

Open Access This article is licensed under a Creative Commons Attribution 4.0 International License, which permits use, sharing, adaptation, distribution and reproduction in any medium or format, as long as you give appropriate credit to the original author(s) and the source, provide a link to the Creative Commons licence, and indicate if changes were made. The images or other third party material in this article are included in the article's Creative Commons licence, unless indicated otherwise in a credit line to the material. If material is not included in the article's Creative Commons licence and your intended use is not permitted by statutory regulation or exceeds the permitted use, you will need to obtain permission directly from the copyright holder. To view a copy of this licence, visit http://creativecommons.org/licenses/by/4.0/.

\section{References}

Aguilera I, Daponte A, Gil F, Hernández AF, Godoy P, Pla A, Ramos JL, On behalf of the DASAHU group (2008) Biomonitoring of urinary metals in a population living in the vicinity of industrial sources: a comparison with the general population of Andalusia. Spain. Sci. Total Environ. 407:669-678

Akesson A, Lundh T, Vahter M, Bjellerup P, Lidfeldt J, Nerbrand C (2005) Tubular and glomerular kidney effects in Swedish women with low environmental cadmium exposure. Environ Health Perspect 113:1627-1631 
Ambak R, He FJ, Othman F et al (2021) Salt intake was higher among males and those with high BMI and waist circumference: introduction to the Malaysian Community Salt Survey (MyCoSS), a population-based salt intake survey in Malaysia. J Health Popul Nutr 40:23. https://doi.org/10.1186/s41043-021-00229-y

Aposhian HV, Aposhian MM (2006) Arsenic toxicology: five questions. Chem Res Toxicology 19:1-15

Aprea MC, Apostoli P, Bettinelli M, Lovreglio P, Negri S, Perbellini L, Perico A, Ricossa MC, Salamon F, Scapellato ML, Iavicolli I (2018) Urinary levels of metal elements in the non-smoking general population in Italy: SIVR study 2012-2015. Toxicol Lett 298:177-185

Arbuckle TE (2006) Are there sex and gender differences in acute exposure to chemicals in the same setting? Environ Res 101:195-204

ATSDR Toxicological profile for cadmium. Department of Health and Human Services, Public Health Service, 2012. Atlanta GA.

Barr DB, Wilder LC, Caudill SP, Gonzalez AJ, Needham LL, Pirkle JL (2005) Urinary creatinine concentrations in the US population: implication for urinary biologic monitoring measurements. Environ Health Perspect 113:192-200

Becker K, Schulz C, Kaus S, Seiwert M, Seifert B (2003) German environmental survey 1998 (GerEs III): environmental pollutants in the urine of the German population. Int J Hyg Env Health 206:15-24

CDC (2005) Third National Report on Human Exposure to Environmental Chemicals. Centers for Disease Control and Prevention, Atlanta, GA

CDC (2009) Fourth National Report on Human Exposure to Environmental Chemicals. Centers for Disease Control and Prevention, Atlanta, GA

CHMS 2013 (Canadian Health Measures Survey Cycle 2) Second Report on Human Biomonitoring of Environmental Chemicals in Canada 2009-2011

Choi BS, Choi SJ, Kim DW, Huang M, Kim NY, Park KS, Kim CY, Lee HM, Yum YN, Han ES, Kang TS, Yu IJ, Park JD (2010) Effects of repeated seafood consumption on urinary excretion of arsenic species by volunteers. Arch Environ Contam Toxicol 58:222-229

Choi J, Knudsen LE, Seher M, Joas A (2017) Identification of exposure to environmental chemicals in children and older adults using human biomonitoring data sorted by age: Results from a literature review. Int J Hyg Environ Health 220:282-298

Cocker J, Mason HJ, Warren ND, Cotton RJ (2011) Creatinine adjustment of biological monitoring results. Occup Med 61:349-353

Cubadda F, Jackson BP, Cottingham KL, Van Horne YO, KurziusSpencer M (2017) Human exposure to dietary inorganic arsenic and other arsenic species: State of knowledge, gaps and uncertainties. Sci Total Environ 579:1228-1239

Department of Statistics Malaysia (DOSM) Current Population Estimates, 2020 https://www.dosm.gov.my/v1/index.php?r=column/ cthemeByCat\&cat=155\&bul_id=OVByWjg5YkQ3MWFZRTN5 bDJiaEVhZz09\&menu_id=L0pheU43NWJwRWVSZk1WdzQ4T lhUUT09 accessed on 2 January 2021

EFSA (2009) Scientific opinion on arsenic in food. EFSA panel on contaminants in the food chain. EFSA J. https://doi.org/10.2903/j. efsa.2009.1351

Ewers U, Krause C, Schulz C, Wilhelm M (1999) Reference values and human biological monitoring values for environmental toxins: Report on the work and recommendations of the Commission on Human Biological Monitoring of the German Federal Environmental Agency. Int Arch Occup Environ Health 72:255-260

FAO (Food and Agriculture Organization) 2018. The State of World Fisheries and Aquaculture -Meeting the sustainable development goals http://www.fao.org/state-of-fisheries-aquaculture accessed online 21 January 2020
Francesconi KA, Kuehnelt D (2004) 2004 Determination of arsenic species: a critical review of method and applications, 2000-2003. Analyst 129:373-395

Ghazali AR, Razak NEA, Othman MS, Othman H, Ishak I, Lubis SH, Mohammad N, Hamid ZA, Harun Z, Kamarulzaman F, Abdullah R (2012) Study of Heavy Metal Levels among Farmers of Muda Agricultural Development Authority. Malaysia Journal of Environmental and Public Health. https://doi.org/10.1155/2012/ 758349

Hariri A, Noor NM, Paiman NA, Zaidi AMA, Bakri SFZ (2018) Heavy metals found in the breathing zone, toenails and lung function of welders working in an air-conditioned welding workplace. Int J Occup Saf Ergon 24(4):646-651. https://doi.org/10.1080/10803 548.2017.1368950

Hoet P, Jacquerye C, Deumer G, Lison D, Haufroid V (2013) Reference values and upper reference limits for 26 trace elements in the urine of adults living in Belgium. Clin Chem Lab Med 51:839-849

International Agency for Research on Cancer (IARC) Some drinking water disinfectants and contaminants, including arsenic. Monographs on the evaluation of carcinogenic risks to humans, Lyon 2002 .

Ismail SNS, Salleh FH, Abidin EZ, Mahiddin NAK, Rangga JU (2018) Cadmium (Cd) Exposure among Waste Collector in Urban Area, Malaysia. Malaysian Journal of Medicine and Health Sciences 14(1):72-80

Jarup L, Akesson A (2009) Current status of cadmium as an environmental health problem. Toxicol Appl Pharmacol 238:201-208

Land M-A, Webster J, Christoforou A et al (2014) Salt intake assessed by $24 \mathrm{~h}$ urinary sodium excretion in a random and opportunistic sample in Australia. BMJ Open 4:e003720. https://doi.org/10. 1136/bmjopen-2013-003720

Lee BK, Kim YH (2014) Sex-specific profiles of blood metal levels associated with metal-iron interactions. Saf Health Work 5:113-117

Lee JW, Lee CK, Moon CS, Choi IJ, Lee KJ, Yi S-M, Jang B-K, Yoon BJ, Kim DS, Peak D, Sul D, Oh E, Im H, Kang HS, Kim JH, Lee J-T, Kim K, Park KL, Ahn R, Park SH, Kim SC, Park C-H, Lee JH (2012) Korea National Survey for Environmental Pollutants in the Human Body 2008: Heavy metals in the blood or urine of the Korean population. Int J Hyg Environ Health 215:449-457

Lin H-T, Chen S-W, Shen C-J, Chu C (2008) Arsenic Speciation in Fish on the Market. J Food Drug Anal 16(4):70-75

López-Herranz A, Cutanda F, Esteban M, Pollán M, Calvo E, PérezGómez B, Cortes MV, BIOAMBIENT.ES, Castaño A (2015) Cadmium levels in a representative sample of the Spanish adult population: The BIOAMBIENT.ES project. J Eposure Sci Environ Epidemiol. https://doi.org/10.1038/jes.2015.25

Mortada WI, Sobh MA, el-Defrawy MM, Farahat SE (2002) Reference intervals of cadmium, lead, and mercury in blood, urine, hair, and nails among residents in Mansoura city, Nile delta. Egypt. Environ Res. 90(2):104-110

Navas-Acien A, Silbergeld EK, Pastor-Barriuso R, Guallar E (2009) Rejoinder: Arsenic exposure and prevalence of type 2 diabetes: updated findings from the National Health Nutrition and Examination Survey, 2003-2006. Epidemiology 20:816-820

Navas-Acien A, Francesconi KA, Silbergeld EK, Guallar E (2011) Seafood intake and urine concentrations of total arsenic, dimethylarsinate and arsenobetaine in the US population. Environ Res 111:110-118

Nigra AE, Sanchez TR, Nachman KE, Harvey DE, Chillrud SN, Graziano JH, Navas-Acien A (2017) The effect of the Environmental Protection Agency maximum contaminant level on arsenic exposure in the USA from 2003 to 2014: an analysis of the National Health and Nutrition Examination Survey (NHANES). Lancet Public Health 2:e513-e521 
Noonan CW, Sarasua SM, Campagna D, Kathman SJ, Lybarger JA, Mueller PW (2002) Effects of exposure to low levels of environmental cadmium on renal biomarkers. Environ Health Perspect 110:151-155

Nurul IA, Rozita WMW, Tengku Rozaina TM, Cheong YL, Siti FD, Nasriyah CH, Nor AA, Rafiza S, Lokman HS (2016) Fish consumption pattern among adults of different ethnics in Peninsular Malaysia. Food Nutr 60:32697

Olsson IM, Bensryd I, Lundh T, Ottosson H, Skerfving S, Oskarsson A (2002) Cadmium in blood and urine impact of sex, age, dietary intake, iron status, and former smoking-association of renal effects. Environ Health Perspect 110:1185-1190

Park C, Hwang M, Kim H, Ryu S, Lee K, Choi K, Paek D (2016) Early snapshot on exposure to environmental chemicals among Korean adults-results of the first Korean National Environmental Health Survey (2009-2011). Int J Hyg Environ Health 219:398-404

Pino A, Amato A, Alimonti A, Mattei D, Bocca B (2012) Human biomonitoring for metals in Italian urban adolescents: data from Latium Region. Int J Hyg Environ Health 215(2):185-190

Schoeters G, Colles A, Den Hon E, Croes K, Vrijens J, Baeyens W, Van De NV, Mieroop E, Covaci A, Bruckers L, Van Larebeke N, Sioen I, Morrens B, Loots I (2012) The Flemish Environment and Health Study (FLEHS) Second Survey (2007-2011): Establishing Reference Values for Biomarkers of Exposure in The Flemish Population. Issues in Toxicology 9:135-165

Schulz C, Wilhelm M, Heudorf U, Kolossa-Gehring M (2011) Update of the reference and HBM values derived by the German Human Biomonitoring Commission. Int J Hyg Environ Health 215:26-35
Steinmaus C, Bates MN, Omar R, Yuan Y (2006) Arsenic Methylation and Bladder Cancer Risk in Case-Control Studies from Argentina and The United States. J Occup Environ Med 48:478-488

Wilhelm M, Ewers U, Schulz C (2004) Revised and new reference values for some trace elements in blood and urine for human biomonitoring in environmental medicine. Int J Hyg Environ Health 207:69-73

World Health Organization (WHO) Biological Monitoring of Chemical Exposure in the Workplace 1996, Vol. 1

Wu MM, Chiou HY, Hsueh YM, Hong CT, Su CL, Chang SF, Huang WL, Wang HT, Wang YH, Huang WL, Wang HT, Wang YH, Hsieh YC, Chen CJ (2006) Effect of plasma homocysteine level and urinary monomethylarsonic acid on the risk of arsenicassociated carotid atherosclerosis. Toxicol Appl Pharmacol 216(1):168-175

Yasuda H, Yoshida K, Yasuda Y, Tsutsui T (2012) Two age-related accumulation profiles of toxic metals. Curr Aging Sci 5:105-111

Zainal Bakri SF, Hariri A, Ismail M, Muhamad Damanhuri AA (2019) Evaluation of Industrial Workplace Exposure to Metal Fumes using Toenail as Bio-Indicator. International Journal of Integrated Engineering 11(5):133-139

Publisher's Note Springer Nature remains neutral with regard to jurisdictional claims in published maps and institutional affiliations. 